\title{
A $200 \mathrm{GHz}$ Monolithic Integrated Power Amplifier in Metamorphic HEMT Technology
}

\author{
I. Kallfass, P. Pahl, H. Massler, A. Leuther, A. Tessmann, S. Koch, and T. Zwick
}

\begin{abstract}
A millimeter-wave monolithic integrated circuit power amplifier operating in the frequency range between 186 and $212 \mathrm{GHz}$ is presented. The amplifier, dedicated to high-resolution imaging radar and communication systems, is realized in a $100 \mathrm{~nm}$ gate length metamorphic high electron mobility transistor technology. The three-stage design with four parallel transistors in the output stage achieves a linear gain of more than $12 \mathrm{~dB}$ and provides a saturated output power of more than $9 \mathrm{dBm}$ and $7 \mathrm{dBm}$ at 192 and $200 \mathrm{GHz}$, respectively.
\end{abstract}

Index Terms-G-band, mHEMT, millimeter-wave field effect transistor (FET) integrated circuits (ICs), millimeter-wave power amplification, monolithic microwave integrated circuits (MMICs).

\section{INTRODUCTION}

A midst today's technologies covering the millimeter-wave $(30-300 \mathrm{GHz})$ frequency range with its applications in remote sensing and communication, the millimeter-wave monolithic integrated circuit (MMIC) offers tremendous advantages in terms of compactness, performance competitiveness as well as ease-of-integration and deployment. The need for high power levels arises predominantly in the transmission paths of active systems, e. g. in high resolution imaging radar and high data rate wireless communication, where operating range is a direct function of the transmit power. In passive systems, heterodyne receivers require high local oscillator power levels to drive their mixers. While MMIC technology has been shown to be capable of providing low-noise amplifiers covering the entire millimeter-wave range [1], [2], relatively few works have been reported on MMIC-based power amplification beyond $100 \mathrm{GHz}$. In [3], Northrop Grumman Corp. (NGC) demonstrated a power amplifier MMIC based on $70 \mathrm{~nm}$ InP high electron mobility

Manuscript received October 16, 2008; revised February 27, 2009. First published May 26, 2009; current version published June 05, 2009. This work was supported by Sony Deutschland GmbH Sensing Systems Laboratory, the German Federal Ministry of Defence (BMVg), and the Bundeswehr Technical Center for Information Technology and Electronics (WTD81).

I. Kallfass, H. Massler, A. Leuther, and A. Tessmann are with the Fraunhofer Institute for Applied Solid-State Physics (IAF), Freiburg D-79108, Germany (e-mail: ingmar.kallfass@iaf.fraunhofer.de).

P. Pahl was with the Fraunhofer Institute for Applied Solid-State Physics (IAF), Freiburg D-79108, Germany and is now with the University of Karlsruhe (TH), Karlsruhe D-7613, Germany.

S. Koch is with Sony Deutschland GmbH Sensing Systems Laboratory, Stuttgart D-70327, Germany.

T. Zwick is with the University of Karlsruhe (TH), Karlsruhe D-7613, Germany.

Color versions of one or more of the figures in this letter are available online at http://ieeexplore.ieee.org.

Digital Object Identifier 10.1109/LMWC.2009.2020042 transistor (HEMT) technology. This amplifier delivers a saturated output power of $13 \mathrm{dBm}$ and $10 \mathrm{dBm}$ up to $190 \mathrm{GHz}$ when operated under a drain supply voltage of $2 \mathrm{~V}$ and $1.2 \mathrm{~V}$, respectively. Entering the sub-millimeter-wave region, NGC recently reported $3 \mathrm{dBm}$ of output power from a three-stage amplifier at $330 \mathrm{GHz}$ [4]. Based on InP double hetero-junction bipolar transistor technology (DHBT) with its high breakdown voltages, a $176 \mathrm{GHz}$ amplifier with $9.1 \mathrm{dBm}$ saturated output power has been realized [5]. In this letter, we report a three-stage power amplifier MMIC based on a $100 \mathrm{~nm}$ metamorphic HEMT technology, achieving an output power of $9 \mathrm{dBm}$ and $7 \mathrm{dBm}$ at 192 and $200 \mathrm{GHz}$, respectively.

\section{MHEMT TECHNOLOGY}

The HEMT based on InGaAs/InAlAs hetero-structures with high In-content in the electron transport channel can be grown either pseudomorphic on InP wafers or by using a metamorphic buffer to adapt the lattice constant on GaAs substrates. Major advantages of the metamorphic approach are cost and quality of the GaAs wafers as well as ease of wafer handling. Disadvantages of the metamorphic HEMT (mHEMT) are the lower thermal conductivity of the metamorphic buffer as well as the additional growth effort. Based on the metamorphic approach, the Fraunhofer Institute for Applied Solid State Physics (IAF) develops advanced MMIC processes including HEMTs with gate lengths from $100 \mathrm{~nm}$ down to $30 \mathrm{~nm}$. Up to an operating frequency of $200 \mathrm{GHz}$ and for power applications, the $100 \mathrm{~nm}$ variant is the most appropriate choice. It features maximum cutoff frequencies $f_{\mathrm{T}}$ of $220 \mathrm{GHz}$ and $f_{\max }$ of $300 \mathrm{GHz}$. On- and off-state breakdown voltages are evaluated to $3.0 \mathrm{~V}$ and $4.3 \mathrm{~V}$, respectively [6].

The mHEMT layers are grown on 4-inch semi-insulating GaAs wafers by molecular beam epitaxy (MBE). For the metamorphic buffer a linear $\mathrm{In}_{x} \mathrm{Al}_{0.48} \mathrm{Ga}_{0.52-x} \mathrm{As}(\mathrm{x}=0 \rightarrow 0.52)$ transition is used. For the $100 \mathrm{~nm}$ gate length technology the electrons are confined in an $\mathrm{In}_{0.65} \mathrm{Ga}_{0.35} \mathrm{As} / \mathrm{In}_{0.53} \mathrm{Ga}_{0.47} \mathrm{As}$ composite channel to increase the breakdown voltages. The low energy electrons with high electron mobility are confined in the $\mathrm{In}_{0.65} \mathrm{Ga}_{0.35}$ As layer. Additionally, the high energy electrons are distributed over both layers, which reduces impact ionization. The split channel is confined by $\mathrm{In}_{0.52} \mathrm{Al}_{0.48}$ As barriers. The upper barrier layer includes a silicon $\delta$-doping. The layer sequence was capped with a highly doped $\mathrm{In}_{0.53} \mathrm{Ga}_{0.47}$ As layer to reduce the ohmic contact and source resistance. The $100 \mathrm{~nm}$ $\mathrm{T}$-gates are defined by a $100 \mathrm{kV}$ electron beam lithography using a three-layer resist. A Pt-Ti-Pt-Au layer sequence was used for the gate metallization. The devices are passivated with a $250 \mathrm{~nm}$ thick CVD deposited $\mathrm{SiN}$ layer, which was 


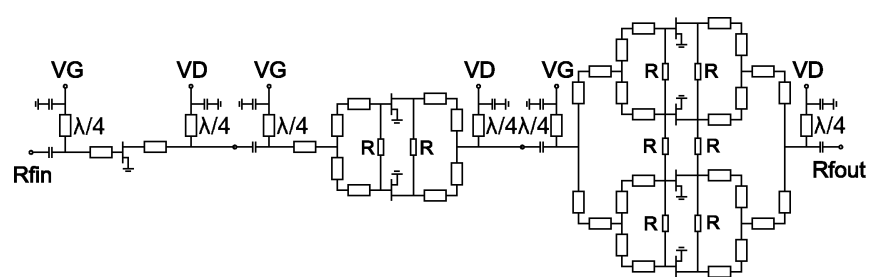

Fig. 1. Circuit schematic of the three-stage power amplifier MMIC.

also used as dielectric layer for the MIM capacitors. Further passive elements are $\mathrm{NiCr}$ thin film resistors, an electron beam evaporated Au based interconnection layer, and a $2.7 \mu \mathrm{m}$ thick plated Au layer in airbridge technology.

For the realization of MMICs operating at frequencies beyond $60 \mathrm{GHz}$ the frontside process is extended by a full backside metallization process, including wafer thinning to $50 \mu \mathrm{m}$ substrate thickness and via hole etching for increased rejection of parasitic substrate modes in the coplanar transmission line environment.

\section{AMPLIFIER DESIGN}

The circuit schematic of the power amplifier is shown in Fig. 1. A three-stage topology is adopted, with the first, second and third stage employing one, two and four common-source transistors in parallel, respectively.

The individual transistors use four gate fingers with $10 \mu \mathrm{m}$ width each, i.e. $4 \times 10 \mu \mathrm{m}$ devices. The transistor size is chosen for maximum size in order to maximize the output power, while maintaining a reasonable small-signal gain of the overall amplifier of at least $10 \mathrm{~dB}$ at $200 \mathrm{GHz}$. The power splitter and combiner networks in front of and after the transistors also serve as matching networks to achieve a $50 \Omega$ interface impedance at every stage's in- and output. The matching networks are designed for maximum gain. Load-pull simulations with largesignal models indicate that further improvement in output power is possible by choosing the appropriate load impedance for maximum output power. The validation of the large-signal model is currently under way.

It is questionable whether the parallelization of transistors is a practicable way for realizing power amplifiers at high millimeter-wave frequencies. The loss incurred by the combining/splitting networks opposes the achievable gain and output power. Our simulations indicate a power loss of approximately $1.2 \mathrm{~dB}$ per combiner/splitter network in the frequency range from 180 to $210 \mathrm{GHz}$. The parallelization of four transistors in the output stage therefore results in a $3.6 \mathrm{~dB}$ increase of the output power from a single transistor. This is paid for by a reduced efficiency, since the DC power consumption scales linearly with the number of parallel transistors.

Shunt resistors at the transistor gate and drain terminals are employed for odd-mode suppression and to ensure stability in parallel amplifier branches. The gate and drain bias voltages are supplied via $\lambda / 4$ lines before and after the power splitter/combiner networks.

Fig. 2 shows the chip photograph of the amplifier MMIC, implemented in a coplanar transmission line environment. Its total chip size is $1 \times 2.25 \mathrm{~mm}^{2}$. The gate and drain supply voltages are

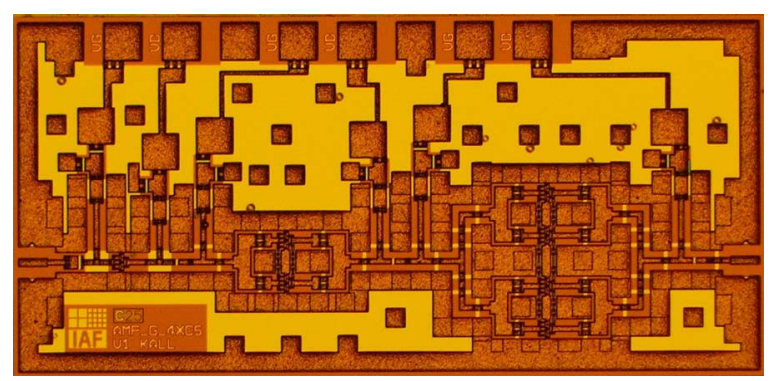

Fig. 2. Chip photograph of the power amplifier MMIC realized in IAF $100 \mathrm{~nm}$ metamorphic HEMT technology.

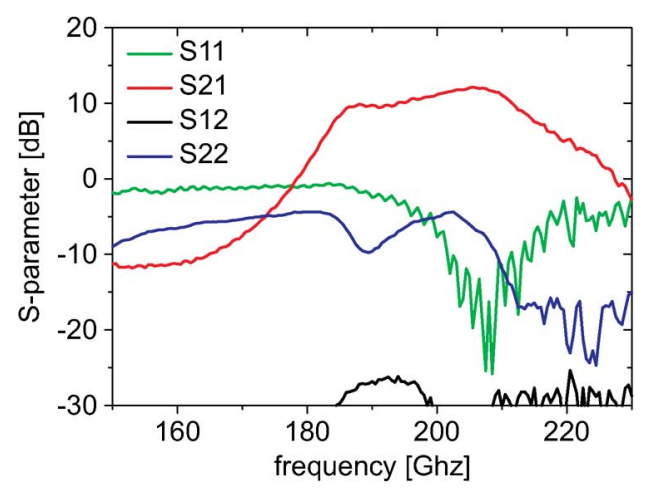

Fig. 3. G-band S-parameter measurement of the $200 \mathrm{GHz}$ power amplifier MMIC.

provided for each stage individually for ease of testing. Special care is taken in the design of the RF shunt capacitance networks to ensure low-frequency stability of the circuit. This is achieved by a deliberate reduction of the shunt capacitors' quality factor.

\section{AMPLIFIER PERFORMANCE}

S-parameter measurements in G-band (140-220 GHz) show the amplifier's linear gain and power matching characteristics. Fig. 3 shows the measured reflection and transmission coefficients. A linear gain of $12.1 \mathrm{~dB}$ is achieved at $205 \mathrm{GHz}$. The 3 -dB bandwidth is $26 \mathrm{GHz}$ (186-212 GHz, corresponding to a relative bandwidth of $13 \%$ ). The bias voltages at the drain and gate are set to $V_{\mathrm{D}}=1.4 \mathrm{~V}$ and $V_{\mathrm{G}}=0.1 \mathrm{~V}$. Under these conditions, the amplifier consumes $160 \mathrm{~mW}$ of DC power and all transistors operate at a current density of $409 \mathrm{~mA} / \mathrm{mm}$.

Output power is measured on-wafer with all power levels normalized to the probe tip reference plane. In order to generate enough input power to drive the amplifier into saturation, a commercial diode-based frequency-doubler module is employed. It can generate up to $5 \mathrm{dBm}$ of output power at the chip input reference plane. The doubler is driven by an IAF power amplifier module delivering more than $100 \mathrm{~mW}$ output power up to $110 \mathrm{GHz}$. The input signal to the power amplifier is generated by a W-band source module. The measurement setup is sketched in Fig. 4.

Fig. 5 shows the measurement results of output power and gain as a function of the input power at 192 and $200 \mathrm{GHz}$. The bias conditions in the power measurement are as stated above. At $192 \mathrm{GHz}$ the amplifier reaches a saturated output power of $9.5 \mathrm{dBm}$ when driven with $5 \mathrm{dBm}$ input power. At $200 \mathrm{GHz}$, 


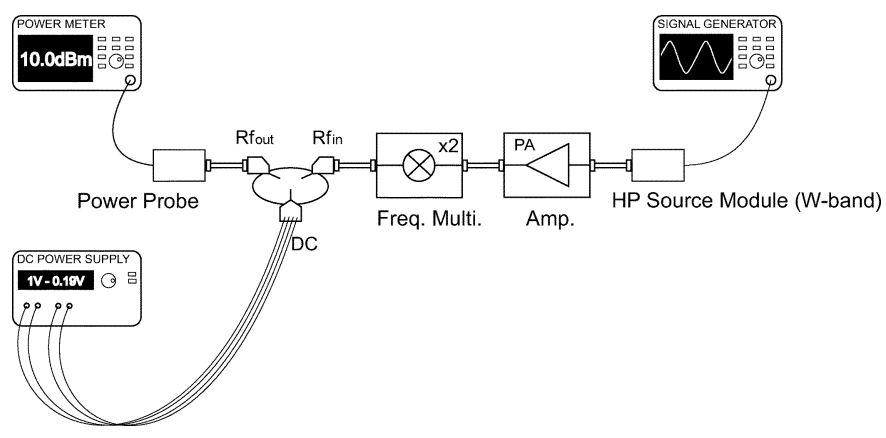

Fig. 4. Output power measurement setup for 200 to $220 \mathrm{GHz}$.

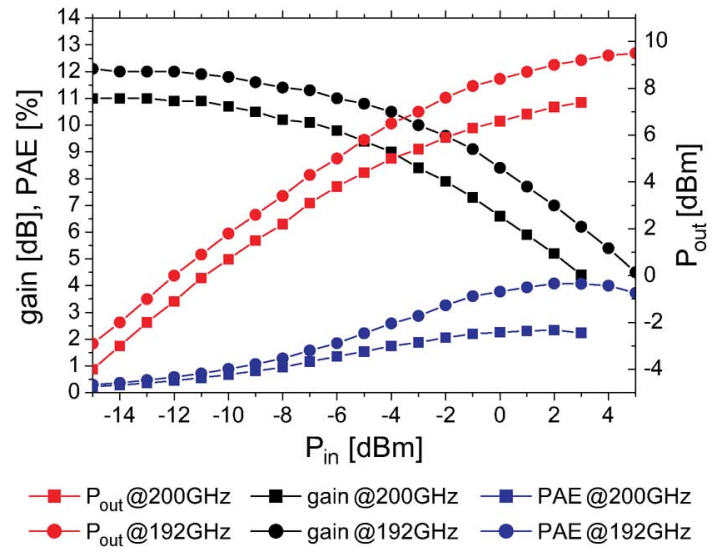

Fig. 5. Output power and gain versus input power at $192 \mathrm{GHz}$ and $200 \mathrm{GHz}$.

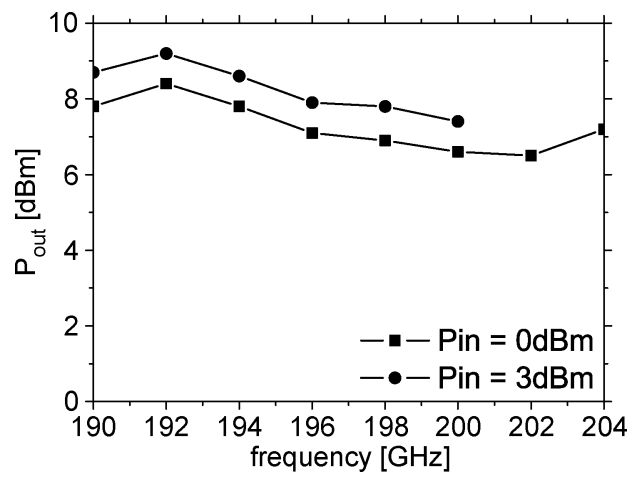

Fig. 6. Measurement of output power in a frequency sweep at constant input power levels.

the output power saturates at $7.4 \mathrm{dBm}$ with a gain of $4.4 \mathrm{~dB}$. The amplifier's output-related $1-\mathrm{dB}$ compression point is at $5.0 \mathrm{dBm}$ and $3.1 \mathrm{dBm}$ for 192 and $200 \mathrm{GHz}$, respectively.

Within the measurement setup constraints, notably the bandwidth of the G-band frequency doubler, a sweep of the frequency was possible between 190 and $205 \mathrm{GHz}$. Fig. 6 shows the amplifier output power for two different input power levels. Within the considered frequency range, no significant power degradation is observed.

Table I shows a comparison of reported power amplifiers in the frequency range from 150 to $300 \mathrm{GHz}$ [7], [8].
TABLE I

COMPARISON OF POWER AMPLIFIERS IN THE FREQUENCY RANGE FROM 150 TO $300 \mathrm{GHz}$

\begin{tabular}{|c|c|c|c|c|c|c|}
\hline Technology & $\begin{array}{c}\text { Frequency } \\
\text { [GHz] }\end{array}$ & $\begin{array}{l}\mathrm{BW} \\
{[\%]}\end{array}$ & $\begin{array}{c}\text { Psat } \\
{[\mathrm{dBm}]}\end{array}$ & $\begin{array}{l}\text { Gain } \\
{[\mathrm{dB}]}\end{array}$ & $\begin{array}{c}\text { PAE } \\
\text { [PAE] }\end{array}$ & Ref. \\
\hline InP HEMT & 260 & 12 & $>5.9$ & 16.5 & 4 & [7] \\
\hline InP HEMT & 270 & - & 7.8 & 12 & 5.25 & [8] \\
\hline InP HBT & 178 & - & 9.1 & 7 & 3 & [5] \\
\hline \multirow{2}{*}{$\begin{array}{c}\text { InP } \\
\text { HEMT }\end{array}$} & 184 & \multirow[t]{2}{*}{8} & $10 @ 1.2 \mathrm{~V}$ & \multirow[t]{2}{*}{20} & 6.5 & \multirow[t]{2}{*}{ [3] } \\
\hline & 184 & & 13@2V & & 9.5 & \\
\hline \multirow{2}{*}{$\begin{array}{c}\text { GaAs } \\
\text { mHEMT }\end{array}$} & 192 & \multirow[t]{2}{*}{13} & 9.5 & \multirow[t]{2}{*}{12.1} & 4 & \multirow[t]{2}{*}{ here } \\
\hline & 200 & & 7.4 & & 2.5 & \\
\hline
\end{tabular}

\section{CONCLUSION}

The $100 \mathrm{~nm}$ metamorphic HEMT technology, which was originally developed and optimized for high cutoff frequencies, can be adopted for the implementation of power amplifier MMICs up to G-band frequencies. Combined with low-noise amplifiers, frequency multipliers and mixers based on the same technology, fully MMIC-based high-performance analog frontends for active radar and communication systems operating in the upper millimeter-wave region become feasible.

\section{ACKNOWLEDGMENT}

The authors wish to thank their colleagues at the Fraunhofer Institute for Applied Solid-State Physics (IAF), for their excellent contributions during epitaxial growth and wafer processing, and $\mathrm{M}$. Schlechtweg and $\mathrm{O}$. Ambacher, for their continuous support throughout this work.

\section{REFERENCES}

[1] W. R. Deal, X. B. Mei, V. Radisic, W. Yoshida, P. H. Liu, J. Uyeda, M. Barsky, T. Gaier, A. Fung, and R. Lai, "Demonstration of a S-MMIC LNA with 16-dB gain at 340-GHz," in Proc. IEEE Compound Semicond. Integr. Circuit Symp. CSICS, Portland, OR, Oct. 2007, pp. 75-78.

[2] A. Tessmann, A. Leuther, H. Massler, W. Bronner, M. Schlechtweg, and G. Weimann, "Metamorphic H-band low-noise amplifier MMICs," in IEEE MTT-S Int. Dig., Honolulu, HI, Jun. 2007, pp. 353-356.

[3] P. Huang, R. Lai, R. Grundbacher, and B. Gorospe, "A 20-mW G-band monolithic driver amplifier using 0.07-um InP HEMT," in IEEE MTT-S Int. Dig., San Francisco, CA, Jun. 2006, pp. 806-809.

[4] W. R. Deal, X. B. Mei, V. Radisic, M. D. Lange, W. Yoshida, P. Liu, J. Uyeda, M. E. Barsky, A. Fung, T. Gaier, and R. Lai, "Development of sub-millimeter-wave power amplifiers," IEEE Trans. Microw. Theory Tech., vol. 55, no. 12, pp. 2719-2726, Dec. 2007.

[5] V. K. Paidi, Z. Griffith, W. Yun, M. Dahlstrom, M. Urteaga, N. Parthasarathy, S. Munkyo, L. Samoska, A. Fung, and M. J. W. Rodwell, "G-band (140-220 GHz) and W-band (75-110 GHz) InP DHBT medium power amplifiers," IEEE Trans. Microw. Theory Tech., vol. 53, no. 2, pp. 598-605, Feb. 2005.

[6] A. Tessmann, "220-GHz metamorphic HEMT amplifier MMICs for high-resolution imaging applications," IEEE J. Solid-State Circuits, vol. 40, no. 10, pp. 2070-2076, Oct. 2005.

[7] W. R. Deal, X. B. Mei, V. Radisic, B. Bayuk, A. Fung, W. Yoshida, P. H. Liu, J. Uyeda, L. Samoska, T. Gaier, and R. Lai, "A new sub-millimeter wave power amplifier topology using large transistors," IEEE Microw. Wireless Compon. Lett., vol. 18, no. 8, pp. 542-544, Aug. 2008.

[8] W. R. Deal, X. B. Mei, V. Radisic, B. Bayuk, A. Fung, W. Yoshida, P. H. Liu, J. Uyeda, L. Samoska, T. Gaier, and R. Lai, "A balanced sub-millimeter wave power amplifier," in IEEE MTT-S Int. Dig., Jun. 2008, pp. 399-402. 